\title{
Increased Perviousness on CT for Acute Ischemic Stroke is Associated with Fibrin/Platelet-Rich Clots
}

\author{
(D)T.R. Patel, (D) S. Fricano, (D) M. Waqas, (D). Tso, (D)A.A. Dmytriw, (D) M. Mokin, (D). Kolega, (D). Tomaszewski, (D)E.I. Levy,
} (D).M. Davies, DK.V. Snyder, (D) A.H. Siddiqui, and (D) V.M. Tutino

\begin{abstract}
BACKGROUND AND PURPOSE: Clot perviousness in acute ischemic stroke is a potential CT imaging biomarker for mechanical thrombectomy efficacy. We investigated the association among perviousness, clot cellular composition, and first-pass effect.

MATERIALS AND METHODS: In 40 mechanical thrombectomy-treated cases of acute ischemic stroke, we calculated perviousness as the difference in clot density on CT angiography and noncontrast CT. We assessed the proportion of fibrin/platelet aggregates, red blood cells, and white blood cells on clot histopathology. We tested for linear correlation between histologic components and perviousness, differences in components between "high" and "low" pervious clots defined by median perviousness, and differences in perviousness/composition between cases that did and did not achieve a first-pass effect.

RESULTS: Perviousness significantly positively and negatively correlated with the percentage of fibrin/platelet aggregates $(P=.001)$ and the percentage of red blood cells $(P=.001)$, respectively. Higher pervious clots had significantly greater fibrin/platelet aggregate content $(P=.042)$. Cases that achieved a first-pass effect $(n=14)$ had lower perviousness, though not significantly $(P=.055)$. The percentage of red blood cells was significantly higher $(P=.028)$ and the percentage of fibrin/platelet aggregates was significantly lower $(P=.016)$ in cases with a first-pass effect. There was no association between clot density on NCCT and clot composition or first-pass effect. Receiver operating characteristic analysis indicated that clot composition was the best predictor of firstpass effect (area under receiver operating characteristic curve: percentage of fibrin/platelet aggregates $=0.731$, percentage of red blood cells $=0.706$, perviousness $=0.668$ ).
\end{abstract}

CONCLUSIONS: Clot perviousness on CT is associated with a higher percentage of fibrin/platelet aggregate content. Histologic data and, to a lesser degree, perviousness may have value in predicting first-pass outcome. Imaging metrics that more strongly reflect clot biology than perviousness may be needed to predict a first-pass effect with high accuracy.

ABBREVIATIONS: AIS = acute ischemic stroke; $A U C=$ area under the curve; $C V=$ coefficient of variation; $F P=$ fibrin/platelet aggregates; $F P E=$ first-pass effect; $\mathrm{MMI}=$ Mattes mutual information; $\mathrm{MT}=$ mechanical thrombectomy; $\mathrm{mTICl}=$ modified $\mathrm{TICl} ; \mathrm{RBC}=$ red blood cells; ROC = receiver operating characteristic; $\mathrm{WBC}=$ white blood cells

C $\mathrm{T}$ is the most common imaging technique used to evaluate patients with acute ischemic stroke (AIS). Together, NCCT and CTA can provide valuable information about the occlusive clot, such as its location, length, and density. ${ }^{1-3}$ One parameter

Received June 2, 2020; accepted after revision August 21.

From the Canon Stroke and Vascular Research Center (T.R.P., S.F., M.W., M.T., J.K., J.T., E.I.L., J.M.D., K.V.S., A.H.S., V.M.T.) and Departments of Mechanical and Aerospace Engineering (T.R.P., V.M.T.), Pathology and Anatomical Sciences (S.F., J.K., J.T., V.M.T.), Neurosurgery (M.W., M.T., E.I.L., J.M.D., K.V.S., A.H.S., V.M.T.), Biomedical Engineering (V.M.T.), and Biomedical Informatics (J.M.D.), University at Buffalo, Buffalo, New York; Department of Medical Imaging (A.A.D.), University of Toronto, Toronto, Ontario, Canada; and Department of Neurosurgery (M.M.), University of South Florida, Tampa, Florida.

Research reported in this publication was supported by the National Center for Advancing Translational Sciences of the National Institutes of Health under award number ULITR001412 to the University at Buffalo (A.H.S., V.M.T.). The content is solely the responsibility of the authors and does not necessarily represent the official views of the NIH. This work was also funded by the Cummings Foundation (V.M.T) derived from these images is clot permeability, or perviousness, which indicates the amount of contrast that diffuses through the clot tissue. ${ }^{1,4}$ Several clinical studies have claimed that perviousness may be an important indicator of how easily occlusive clots can be treated by tPA or mechanical thrombectomy (MT). ${ }^{1,5}$ However, the underlying biology behind why some clots are more pervious than others remains largely unknown.

Data are available on reasonable request. The data are composed of deidentified participant data and can be obtained upon request from Dr Vincent Tutino at vincentt@buffalo.edu.

Please address correspondence to Vincent M. Tutino, PhD, 875 Ellicott St, Canon Stroke and Vascular Research Center, University at Buffalo, Buffalo, NY 14203; e-mail: vincentt@buffalo.edu

--.- Indicates open access to non-subscribers at www.ajnr.org

Indicates article with supplemental online photos.

http://dx.doi.org/10.3174/ajnr.A6866 
The advent of MT for the treatment of AIS has enabled the biologic investigation of retrieved clots by histopathology. ${ }^{6}$ Recent studies have shown large-scale heterogeneity in the composition of retrieved clots, in terms of the percentage of fibrin/platelet aggregates (FP), red blood cell (RBC), and white blood cells (WBC). ${ }^{7}$ Differences in clot composition have been associated with stroke etiology and MT treatment outcomes. In several studies, FP-rich clots have been found to be cardioembolic in origin ${ }^{8-10}$ and less responsive to revascularization attempts. ${ }^{6,10,11}$ Intrinsically, perviousness is likely to be related to the cellular composition of a clot because different biologic components have different densities and thus alter how soluble molecules diffuse through them. ${ }^{4,5}$ Experimental studies have shown that thrombi composed of higher fibrin fractions allowed greater contrast agent permeation than those packed with RBC. ${ }^{12}$ Nevertheless, this association has not been established in clinical studies.

To this end, the goal of this study was to investigate the relationship between clot perviousness calculated at the time of admission CT and its histopathologic composition after retrieval by MT. Furthermore, to explore whether perviousness could be used to indicate MT effectiveness, we also explored the relationships among perviousness, composition, and MT outcome, measured by the first-pass effect (FPE). ${ }^{13}$ Characterization of the occlusive clot based on pretreatment NCCT/CTA could impact the clinical management of patients with AIS.

\section{MATERIALS AND METHODS \\ Clinical Study Parameters}

This study was approved by the institutional review board at the University at Buffalo (study 00002092). All methods were performed in accordance with the approved protocol, and written informed consent was obtained for all subjects. Clot samples and imaging were collected from patients receiving MT by either stent retriever, aspiration, or a combined therapy between November 2018 and November 2019 at the Gates Vascular Institute in Buffalo, New York. Patients considered for this study had the following criteria: 1) They had undergone MT for AIS due to an occlusive clot in the intracranial vasculature, 2) had pretreatment CT available with sufficient image quality and an identifiable clot, and 3) had a clot of sufficient size and quality for histopathologic analysis that was retrieved during MT. Cases were excluded for the following reasons: 1) They were lacking pretreatment CT, 2) had CT with insufficient quality (ie, unidentifiable clot), or 3) received intra-arterial tPA. Treatment success was assessed on DSA using the modified TICI (mTICI) score, ${ }^{14}$ which is based on the degree of reperfusion in the downstream territory of the original occlusion: mTICI $2 b$ (50\%-89\% reperfusion), mTICI 2c (90\%-99\% reperfusion), or mTICI 3 (100\% reperfusion). First-pass effect was defined as having achieved mTICI scores of $2 c$ or 3 at the end of the first use of MT. ${ }^{13}$ Modified FPE was further defined as an mTICI score of $2 b, 2 c$, or 3 after the first MT pass. ${ }^{13}$

\section{CT}

For all patients, CT was performed on an Aquilion ONE scanner (Canon Medical Systems). The tube voltage and tube current were set to $135 \mathrm{kV}$ (peak) and 370-600 mA, respectively, for NCCT, and $120 \mathrm{kVp}$ and $150-205 \mathrm{~mA}$, respectively, for CTA.
The reconstructed voxel sizes for NCCT and CTA were set to $0.4 \times 0.4 \times 0.5 \mathrm{~mm}^{3}$ and $0.5 \times 0.5 \times 0.5 \mathrm{~mm}^{3}$, respectively. For CTA, Omnipaque 350 contrast agent (iohexol; GE Healthcare) was given at a rate of $\sim 5 \mathrm{~mL} / \mathrm{s}$ in CT perfusion during image acquisition. The CTA image with the lowest thickness $(0.5 \mathrm{~mm})$ and highest resolution was selected for analysis of the clot region.

\section{Analysis of CT}

Analysis of CT was blinded to clinical outcomes and clot composition. For clots that were hyperdense on NCCT images, no image registration techniques were used, and the CTA and NCCT images were placed side-by-side and Hounsfield units were calculated in the clot region on each image. For clots that were not hyperdense on NCCT, the CTA and the corresponding NCCT images were coregistered using the open source software, 3D Slicer (https://www. slicer.org/), which implemented the BRAINSFit algorithm (https:// www.slicer.org/wiki/Modules:BRAINSFit) for mutual-information rigid registrations of whole-brain $3 \mathrm{D}$ images. ${ }^{15}$ During coregistration, we used the following input parameters: rigid $(d f=6), 0.2 \%$ of samples (percentage of sampled pixels, high) and linear interpolation (to scale image size). After each coregistration, the Mattes mutual information (MMI), a quantitative measure of how similar the coregistered images are (higher MMI implies greater entropy reduction and better image alignment), was recorded and the coregistered images were inspected to ensure proper overlay. See Online Fig 1 for an example of coregistration. The average MMI across all 11 coregistered nondense cases was $92.2 \% \pm 1.1 \%$, with a range of $85.2 \%$ $98.7 \%$, showing highly accurate coregistration across all cases. After coregistration, the occlusion on the CTA image was used to identify the clot in the corresponding location on the NCCT image, and the Hounsfield units (averaged from 2-3 regions) were obtained from both NCCT and CTA images.

As shown in Fig $1 A$, perviousness was calculated as the difference in Hounsfield units, ${ }^{16}$ in which larger differences indicate higher permeability. Cases were categorized as "high" or "low" perviousness based on a cutoff of the median perviousness across all cases in the dataset. Because clot density on NCCT may be independently associated with clot biology and FPE, we also recorded density on NCCT and categorized cases as high or low density based on a cutoff of the median density across all cases in the dataset.

We assessed the inter- and intrauser error in calculating clot perviousness and density in a subset of 10 representative cases (3 dense and 7 nondense; numbers chosen to maintain proportionality to entire dataset). The perviousness and density calculations were performed by the same trained operator in triplicate to calculate intrauser error in the measurement and were performed once by 2 additional trained operators to calculate interuser error among the 3 operators. The average intrauser error (with coefficient of variation $[\mathrm{CV}]$ ) in perviousness and density on NCCT for dense (error perviousness: $\pm 1.6 \mathrm{HU}[10.40 \%], \mathrm{CV}=0.18$; error $_{\text {density: }} \pm 1.6 \mathrm{HU}$ [2.79\%], CV $=0.048$ ) and nondense (error $_{\text {perviousness: }} \pm 1.8 \mathrm{HU}[17.52 \%], \mathrm{CV}=0.30$; error $_{\text {density }}$ : $1.0 \mathrm{HU}$ [1.66\%], CV $=0.029)$ clots was low. The average interuser error in perviousness and density for the dense (error $_{\text {perviousness: }} \pm 1.8 \mathrm{HU}[10.75 \%], \mathrm{CV}=0.19$; error $_{\text {density }}$ :

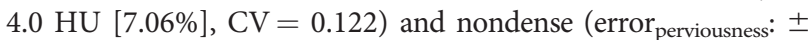
$1.6[12.10 \%], \mathrm{CV}=0.21$; error $_{\text {density: }} \pm 2.0 \mathrm{HU}[3.38 \%], \mathrm{CV}=$ 


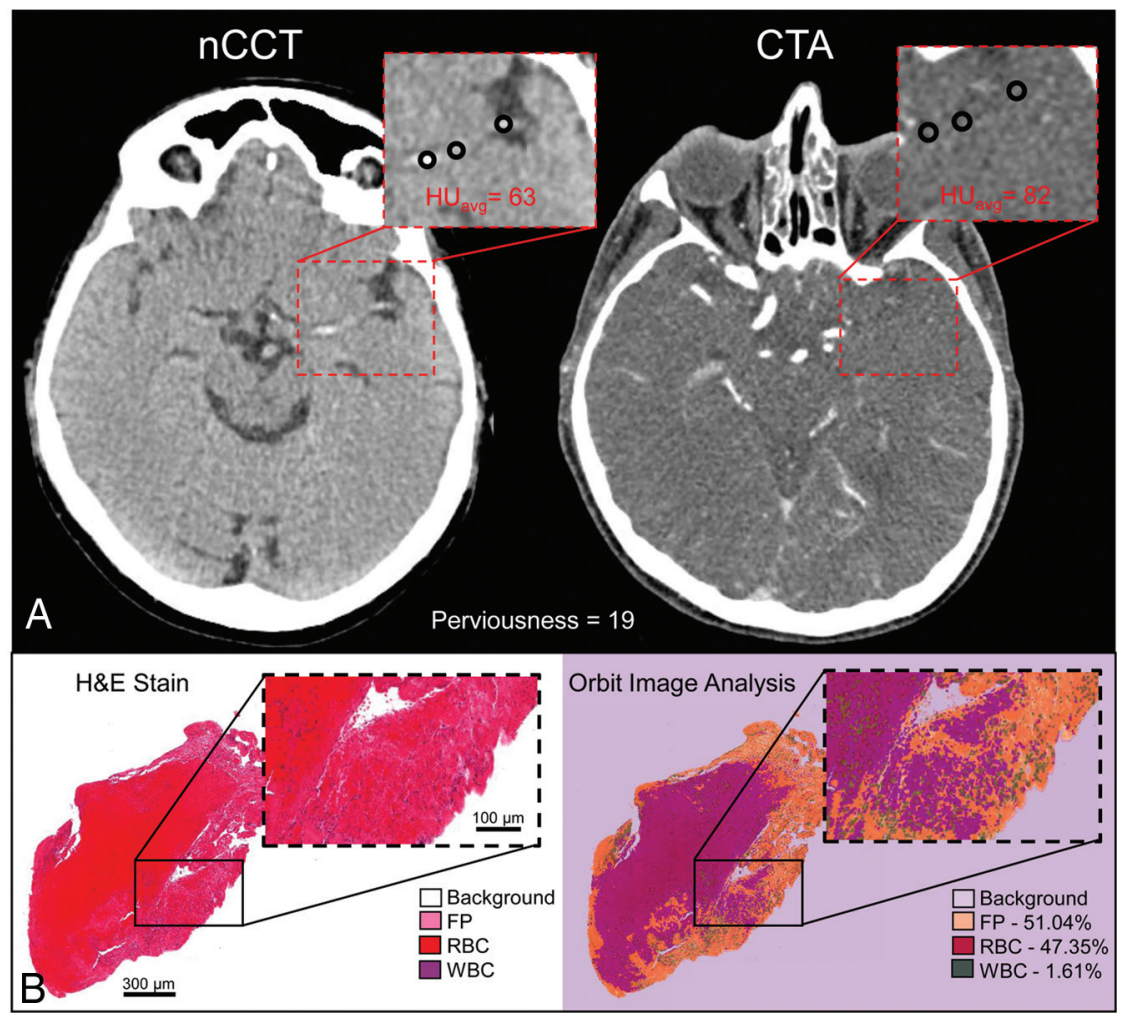

FIG 1. Data acquisition: calculation of perviousness and clot composition. A, Perviousness was calculated from the difference in Hounsfield units in the clot region between CTA and NCCT. B, To identify the percentage composition of major clot components, we analyzed H\&E-stained slides (left) with Orbit Image Analysis (right, colorized output) to automatically identity \%FP, \%RBC, and \%WBC.

\section{Quantification of Clot Composition}

Quantification of clot composition was performed on digital histology images using the open source software, Orbit Image Analysis (www. orbit.bio) (Fig 1B). ${ }^{17}$ Before quantification, all slide images were adjusted to be of the same resolution, and errors in slide preparation (ie, small folds or debris) were manually removed from the image. Next, the Orbit Image Analysis software trained machine learning algorithms for image segmentation, classification, and quantification using a support vector machine based on a sliding window feature-extraction approach. ${ }^{17}$ The models for classification and quantification of histology were adjusted for inter- and intraslide variability in $\mathrm{H} \& \mathrm{E}$ staining by manually marking regions of $\mathrm{RBC}, \mathrm{WBC}$, and FP aggregates as representative regions for the model to be retrained/ adjusted. Once trained, these models were applied to the entire slide, classifying each pixel. This pipeline was used to identify regions of $\mathrm{FP}, \mathrm{RBC}$, and $\mathrm{WBC}$ and to calculate their percentage across each slide.
0.059) clots was also low. There was no significant difference in error or CV between dense and nondense measurements and between intra- and interuser measurements (all $P<.05$, Student $t$ test), showing good user agreement and reproducibility.

\section{Clot Histology}

For histologic analysis, clots were collected immediately after MT and stored in $10 \%$ phosphate buffered formalin for 24-28 hours, after which they were transferred to $70 \%$ ethanol. In the Histology Core Lab at the University at Buffalo's Jacobs School of Medicine and Biomedical Sciences, tissue was automatically prepared in a tissue processor, where it was serially infused with $70 \%$ ethanol, $90 \%$ ethanol, 100\% ethanol, xylenes, and paraffin. All steps were performed under pressure to ensure proper infusion. Samples were then embedded in paraffin and sectioned at $4 \mu \mathrm{m}$. Sections were collected on SuperFrost Plus (Fisher) slides to ensure tissue adhesion. For H\&E staining, samples were deparaffinized in xylene, incubated in serial solutions of ethanol (100\%$50 \%$ ), and hydrated in water. Next, they were incubated in hematoxylin for 7-8 minutes and washed and incubated in eosin for 12 minutes. Stained sections were then dehydrated in graded ethanol, cleared in xylenes, and mounted on a glass microscope slide with a xylene-based mounting medium. Stained slides were imaged by whole-slide scanning at $40 \times$ on the ImageScope digital histology platform (Leica Biosystems).

\section{Statistical Analyses}

All analysis was performed in Matlab (Version 2020a; MathWorks). Pearson correlation tests were performed to assess the correlation between 2 continuous variables. We used univariate tests to assess significant differences among groups. Sharpiro-Wilk tests were performed to test parameters for normality. To test parameters for significance, we performed a Student $t$ test (for normally distributed parameters) or a Mann-Whitney U test (for non-normally distributed parameters). Data values were reported as mean \pm standard error. To test significant differences between 2 categoric variables, we performed a $\chi^{2}$ test. A parameter was deemed significant at $P<.05$.

\section{RESULTS}

\section{Patient Characteristics}

A total of 40 patients with AIS were included in this study. Clinical parameters, treatment methods, mTICI scores, and pre- and posttreatment NIHSS score are summarized in the Table. Occlusions were primarily located at the MCA (67.5\%), supraclinoid ICA $(20 \%)$, and basilar artery (12.5\%) and were treated with stent retrievers $(2.5 \%)$, direct aspiration alone (12.5\%), or a combination therapy (85\%). A total of 14 of the 40 (35\%) cases achieved FPE (21/40 [(52.5\%] cases achieved a modified FPE). The average density on NCCT and CTA images for clot regions was $59.42 \pm 0.88$ $\mathrm{HU}$ and $85.40 \pm 2.31 \mathrm{HU}$, respectively. The corresponding perviousness values ranged from 5 to 62, with an average perviousness 


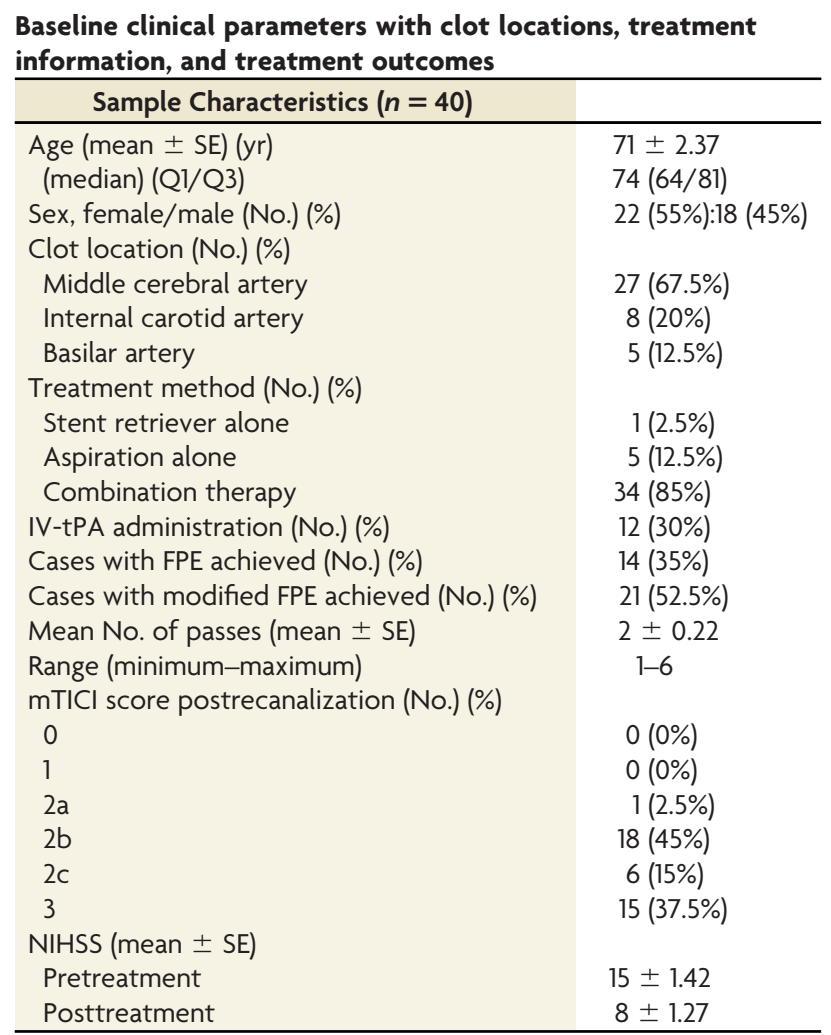

Note:-Q indicates quartile; SE, standard error.

of $26.38 \pm 2.26 \mathrm{HU}$. On the basis of the median perviousness (23.5 $\mathrm{HU})$, we delineated "lower" perviousness clots as those with $<23.5$ HU, and "higher" perviousness clots as those with $>23.5 \mathrm{HU}$. This cutoff system was markedly similar to others reported in the literature; eg, Santos et $\mathrm{al}^{18}$ found an optimal perviousness cutoff of $\geq 23 \mathrm{HU}$ to define high-pervious clots in a dataset of 308 patients with AIS treated with tPA. For analysis of clot density on NCCT, we also used the median clot density from NCCT (60 HU) to independently define low-density clots as those with $<60 \mathrm{HU}$ and high-density clots as those with $>60 \mathrm{HU}$.

\section{Clot Composition Was Correlated with Clot Perviousness but Not Density}

We first explored the relationship between clot presentation on $\mathrm{CT}$ and composition. For all clots, the average percentage composition of $\mathrm{FP}, \mathrm{RBC}$, and $\mathrm{WBC}$ was $50.62 \% \pm 3.83 \%$, $43.43 \% \pm 3.81 \%$, and $5.95 \% \pm 0.50 \%$, respectively. As shown in Fig $2 A-C$, Pearson correlation analysis revealed a significant positive linear correlation between the $\% \mathrm{FP}$ and perviousness $(r=0.496, P=0.001)$ and a significant negative linear correlation between $\% \mathrm{RBC}$ and perviousness $(r=-0.491, P=0.001)$. No significant relationship between \%WBC and perviousness was observed ( $r=-0.057, P=0.726)$. Conversely, there was no significant correlation between clot density on NCCT and clot composition (\%FP, $r=-0.004, P=0.978$; \%RBC: $r=0.009$, $P=0.955$; \%WBC: $r=-0.036, P=0.825)$ (Fig 2D-F).

Comparing composition between clots with higher and lower perviousness in Fig $3 A$, we found that the $\% \mathrm{FP}$ in higher perviousness clots $(54.63 \% \pm 5.76 \%)$ was statistically significantly greater than that in lower perviousness clots $(39.87 \% \pm 4.00 \%)(P=.042)$. On the other hand, the \% $\mathrm{RBC}$ in clots with higher perviousness
$(38.40 \% \pm 5.72 \%)$ was statistically significantly lower than in those with lower perviousness $(53.23 \% \pm 3.99 \%)(P=.040)$. The difference in \%WBC between the high $(6.97 \% \pm 0.55 \%)$ and low $(6.90 \% \pm$ $0.62 \%)$ perviousness groups was not statistically significant $(P=.936)$. When density on NCCT alone was considered, differences in biologic composition between higher and lower density clots were less pronounced. Higher density clots had greater \%RBC $(50.67 \% \pm 4.55 \%$ versus $45.26 \% \pm 5.58 \%, P=.493)$ and $\% \mathrm{WBC}$ $(7.63 \% \pm 0.62 \%$ versus $6.27 \% \pm 0.57 \%, P=.147)$ and lower $\% \mathrm{FP}$ $(41.70 \% \pm 4.34 \%$ versus $48.47 \% \pm 5.65 \%, P=.388)$, but the differences were not statistically significant (Fig 3B).

Because some patients in the preceding analysis had received IV-tPA during the first 4.5 hours after symptom onset, but before imaging, we tested whether the administration of IV-tPA affected our observations. We again compared \%FP, \%RBC, and \%WBC in high-versus-low perviousness and density clots, this time separating cases that were given IV-tPA from those that were not. These data showed that the trend of greater \%FP and lesser \%RBC in higher perviousness clots was still evident regardless of IV-tPA administration. However, some differences did not reach statistical significance, likely due to the decreased sample size in these small subcohorts (Online Fig 2).

\section{First-Pass Effect is Correlated with Clot Composition, but Not Perviousness}

We also explored the relationship between clot presentation on CT and FPE and observed lower perviousness in cases that achieved FPE $(20.50 \pm 2.82 \mathrm{HU}$ versus $29.58 \pm 2.98 \mathrm{HU}$ in cases that did not achieve FPE, Fig $4 A$ ). However, this difference was not statistically significant $(P=.055)$. There was no significant difference in clot density on NCCT between cases that did $(59.64 \pm 1.74 \mathrm{HU})$ and did not achieve FPE $(59.31 \pm 1.09 \mathrm{HU}, P=.859)$. On the other hand, we did observe that the \%FP on histology was statistically significantly lower in cases that achieved FPE $(38.25 \% \pm 4.45 \%)$ compared with those that did not $(57.29 \% \pm 4.95 \%)(P=.016)($ Fig $4 B)$. Furthermore, \%RBC on histology was statistically significantly higher in cases that achieved FPE $(54.69 \% \pm 4.41 \%)$ compared with cases that did not $(37.36 \% \pm 5.01 \%)(P=.028)$. The difference in $\%$ WBC between cases that did $(7.06 \% \pm 0.71 \%)$ and did not achieve FPE $(5.35 \% \pm 0.64 \%)$ was not statistically significant $(P=.103)$. On the basis of these data, post hoc power analysis demonstrated an average power of 0.78 in detecting differences in these significant parameters $(\% \mathrm{FP}$, power $=0.82 ; \% \mathrm{RBC}$, power $=0.74)$ given our sample size, and an $\alpha=.05$. Analogous results were also observed when modified FPE was considered (Online Fig 3).

To explore whether IV-tPA administration affected these observations, we performed our analysis on the subcohorts that either were and were not given IV-tPA. Yet again, similar trends were observed regardless of IV-tPA administration. The most significant differences were seen in the subcohort with IV-tPA administration (cases without IV-tPA administration showed similar trends but no statistically significant differences in these parameters between cases with and without FPE) (Online Fig 4).

To determine predictive ability of perviousness and histologic parameters in assessing FPE, we performed receiver operating characteristic (ROC) analysis on our data. The ROC curves in Fig 4 show that $\% \mathrm{FP}$ and $\% \mathrm{RBC}$ were the best predictors of $\mathrm{FPE}$ 

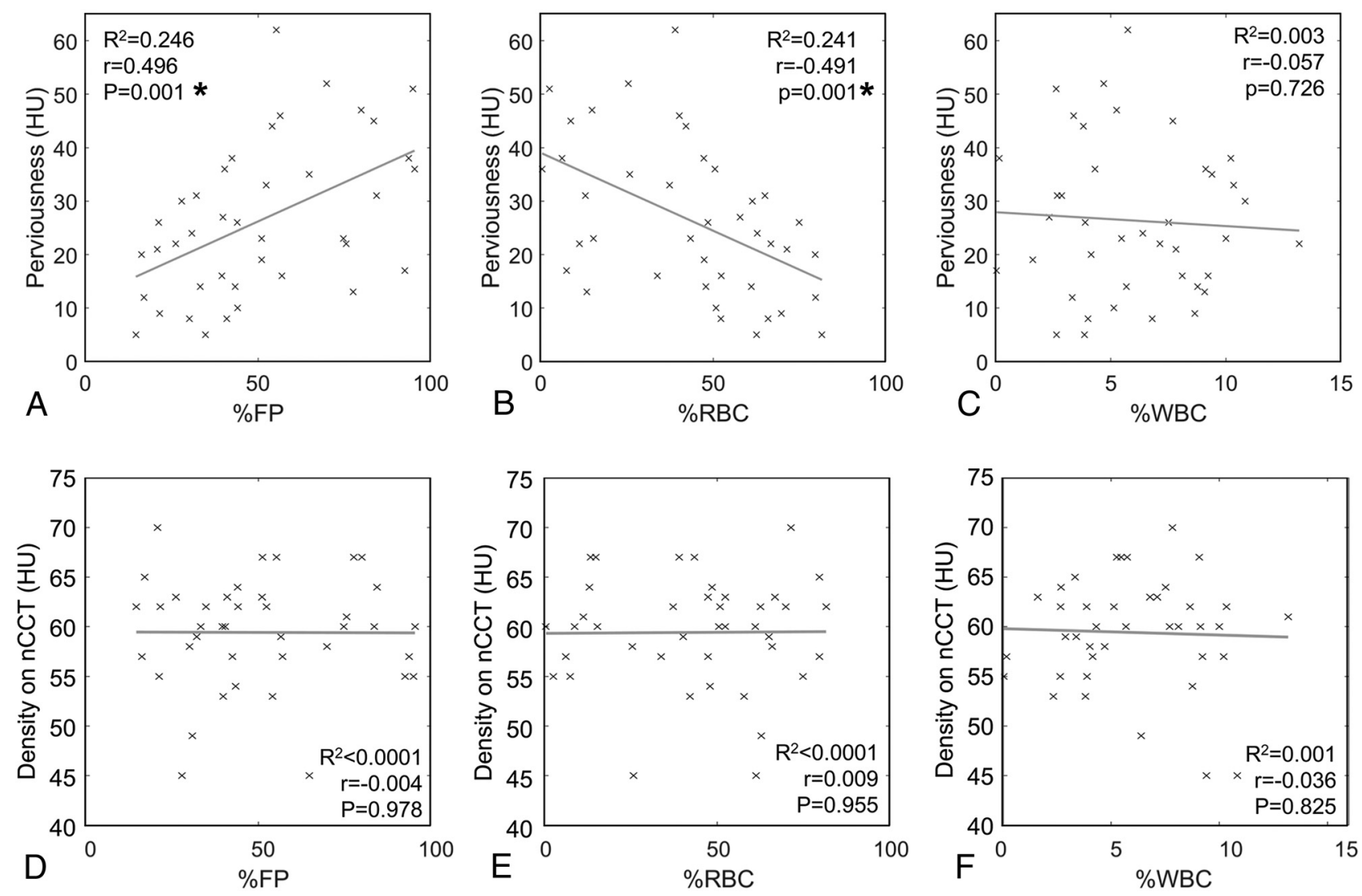

FIG 2. Correlation of perviousness and density with clot biology. A, Pearson correlation of perviousness and \%FP shows a statistically significantly positive association. $B$, Pearson correlation of perviousness and \%RBC shows a statistically significantly negative association. $C$, Pearson correlation of perviousness and \%WBC shows no significant relationship. D, Pearson correlation of density on NCCT and \%FP shows no association. E, Pearson correlation of density on NCCT and \%RBC shows no association. F, Pearson correlation of density on NCCT and \%WBC shows no association. The asterisk indicates statistical significance $(P<0.05)$.
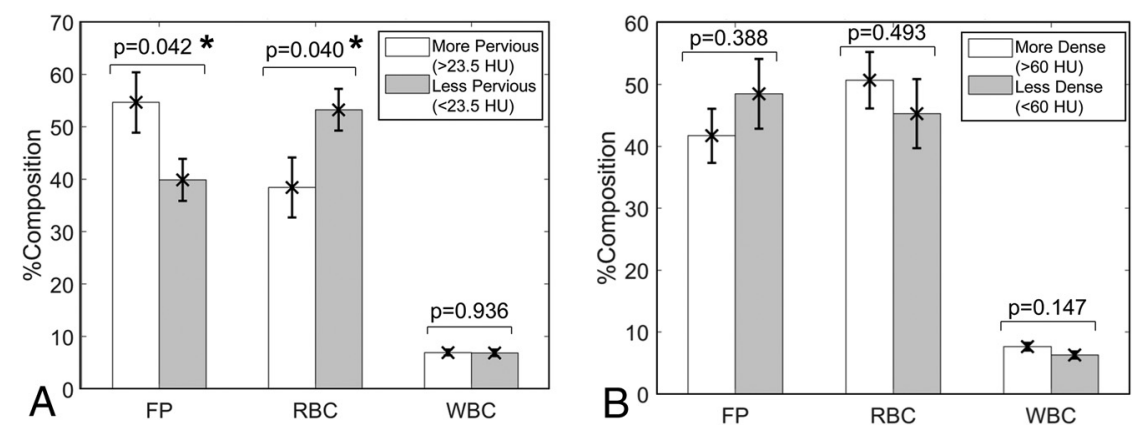

FIG 3. Difference in clot biology in high-versus-low perviousness and density clots. $A$, Significantly greater \%FP and lower \%RBC were evident in higher pervious clots $(>23.5 \mathrm{HU}, n=20)$ compared with lower pervious clots $(<23.5 \mathrm{HU}, n=20)$. $B$, There was no significant difference in \%FP, \%RBC, or $\% \mathrm{WBC}$ in higher density clots $(>60 \mathrm{HU})$ compared with lower density clots $(<60 \mathrm{HU})$. The asterisk indicates statistical significance $(P<0.05)$.

(\%FP: area under the curve $[\mathrm{AUC}]=0.731, \% \mathrm{RBC}: \mathrm{AUC}=0.706$ ), while perviousness on NCCT/CTA had moderate predictive ability $($ AUC $=0.668)$. Clot density on NCCT performed inferior to all other metrics with an AUC $=0.571$. Similar results were observed when modified FPE was used as the predictive end point (Online Fig 3).

\section{DISCUSSION}

In this preliminary study, we explored the utility of perviousness as a pre-MT imaging parameter by investigating the relationship among perviousness, clot composition, and MT treatment effectiveness (ie, FPE). Our results demonstrated a statistically significant correlation between perviousness and clot composition, with higher perviousness associated with greater \%FP. There was no significant correlation between clot composition and density measured on NCCT. Furthermore, we found that clots from cases that achieved FPE had statistically significantly higher \%RBC and lower \%FP. From ROC analysis, \%FP and \%RBC on histology and (to a lesser extent) perviousness on imaging potentially had value in predicting FPE by MT.

In exploring the relationship between clot composition and perviousness, we found that the \%FP was significantly positively correlated with perviousness. On the basis of a 23.5-HU (median 

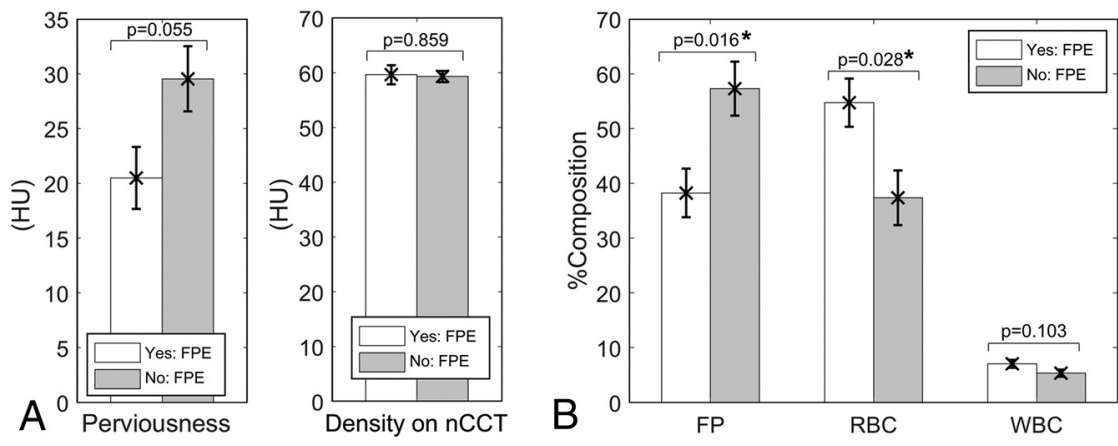

perform Pearson correlation analysis to assess the relationship between perviousness and clot composition in our study in an unbiased manner.

The relationship between increased perviousness and increased \%FP has also been shown in in vitro reports. Borggrefe et $\mathrm{al}^{12}$ explored thrombus composition of histologically-defined ovine blood clots with NCCT and CTA using spectral-detector CT and found that higher FP content was an independent predictor of the increase in thrombus density (ie, perviousness). ${ }^{12}$ They speculated that this phenomenon occurred because fibrin proteins can have an intrinsic affinity to iodine, as shown in several benchtop studies. ${ }^{22,23}$ Their affinity to iodine could cause them to retain contrast media and therefore exhibit higher perviousness. Alternatively, the observed relationship may exist because tightly-packed RBC (that have impenetrable cell walls and may exist in dense rouleaux) can be trapped in fibrin filaments and inhibit blood flow, ${ }^{24,25}$ while porous fibrin meshes can allow contrast agent to penetrate into the clot more easily. ${ }^{8,26}$ Indeed, this difference could be a reason why higher perviousness has been related to treatment success by tPA

perviousness) cutoff, we also found statistically significantly higher $\% \mathrm{FP}$ and lower \%RBC in those with higher perviousness compared with clots with lower perviousness. This trend seemed to exist independent of whether the patient had previously received IV-tPA. These results are similar to those published by Berndt et al, ${ }^{19}$ who also investigated the relationship between MT-retrieved clots and perviousness on NCCT/CTA. On the basis of Pearson correlation analysis, they also found that the \%FP and the \%RBC were positively and negatively related to perviousness, respectively. Remarkably, their correlation coefficients $(\mathrm{FP} \propto$ perviousness $=$ 0.45 ; RBC $\propto$ perviousness $=-0.49$ ) were patently similar to ours $(\mathrm{FP} \propto$ perviousness $=0.50 ; \mathrm{RBC} \propto$ perviousness $=-0.49$ ).

Only 1 other study has explored the relationship between clot composition and perviousness in MT-treated patients with AIS. In a study of $n=57$ patients, Benson et $\mathrm{al}^{20}$ dichotomized both perviousness and clot composition (RBC-rich versus FP-rich) and found that the pervious group consisted of a higher fraction of clots in the RBC-rich group. This contradictory result is likely because unlike in our study and that of Berndt et $\mathrm{al}^{19}{ }^{19}$ Benson et $\mathrm{al}^{20}$ did not directly investigate the association between clot composition and perviousness (both of which are continuous variables). Due to their statistical methods, it is difficult to reliably interpret these findings. One must be careful when dichotomizing variables, especially with both independent and dependent variables as in Benson et al, ${ }^{20}$ because dichotomization is known to reduce statistical power and increase the risk of false-positive results. ${ }^{21}$ Thus, we chose to first in several studies, because increased fluid penetration into the clot may bolster the effectiveness of tPA.,18

Another goal of our study was to determine the relationship between FPE and clot composition and perviousness. Our data show that cases of FPE had significantly more \%RBC than those that did not achieve FPE, which, in turn, had significantly greater $\%$ FP. This trend was also evident in both subcohorts of patients who did and did not receive IV-tPA. In the clinical literature, it has been widely demonstrated that fibrin-rich clots are more difficult to retrieve (ie, take multiple passes) than RBC-rich clots. ${ }^{11,27}$ Poorer MT device integration in FP-rich thrombi may be due to the high static coefficient of friction, as demonstrated by Gunning et $\mathrm{al}^{28}$ This can result in increased adherence to the vessel wall and decreased compressibility of fibrin-rich clots. ${ }^{20}$ Conversely, high RBC density has been associated with successful recanalization after thrombectomy and reduced thrombectomy procedure time. ${ }^{27}$

While clot biology was significantly related to FPE, we did not find a statistically significant association between perviousness and FPE, even though perviousness and clot composition were correlated with each other. This result may be because of their weak relationship seen on Pearson correlation analysis, which could lead to information loss when inferring clot composition from perviousness. ${ }^{12}$ It is possible that a larger sample size would find a more robust correlation. Studies on the relation between clot perviousness and FPE remain scant; only 1 report, Byun et $\mathrm{al}^{29}{ }^{29}$ compared FPE with CT parameters across 58 cases of AIS treated by stent retrievers. They also 
demonstrated that perviousness did not predict FPE or overall successful recanalization. However, even though it was not significant on univariate examination, our ROC analysis demonstrated that perviousness had moderate ability in predicting FPE (AUC $=0.668$ ). Thus, we postulate that other, more informative parameters or combinations of parameters (ie, clot length, ${ }^{2}$ device angulation, ${ }^{30}$ or higher-level radiomics features ${ }^{31}$ ) that more closely reflect the biology or morphology of the occlusive clot could provide better prediction of FPE from pretreatment imaging.

In addition to perviousness, we also investigated the relationship between clot density on NCCT and clot composition and FPE in this study. Unlike perviousness, Pearson correlation analysis did not show any linear relationship between density and biologic composition. We did, however, observe that higher density clots tended to have greater $\% \mathrm{RBC}$, albeit the difference was not statistically significant. This trend echoes other findings in the literature, which have demonstrated that increased \% RBC is associated with hyperdensity on CT imaging. ${ }^{12,25,32}$ Similar to perviousness, clot density on NCCT showed no statistically significant difference between cases that did and did not achieve FPE. Yet on ROC analysis, density had a much lower AUC than perviousness, suggesting that density has little predictive ability for FPE. These results are similar to the report by Jagani et $\mathrm{al},{ }^{33}$ who studied $n=80$ cases of MT treated with aspiration, a stent retriever, or a combination therapy and found no association between clot density and successful recanalization. Another study from the Multicenter Randomized Clinical Trial of Endovascular Treatment for Acute Ischemic Stroke in the Netherlands (MR CLEAN) Registry Investigators ${ }^{34}$ investigated a total of 408 patients with AIS, calculating a gamut of imaging parameters such as location, clot burden score, thrombus length, and clot density and found that clot density was not significantly associated with functional outcome measured using the mRS, degree of reperfusion (TICI), or duration of endovascular treatment. However, at least 1 other study has demonstrated a significant association between density and first-pass outcome. ${ }^{35}$ Thus, further research in larger datasets is likely required to elucidate the relationship between perviousness and density and MT effectiveness measured by FPE.

Our study has limitations. First, to increase our sample size, we did not control for some variables that may affect our results. Specifically, we included cases of MT that used all endovascular approaches and cases that both did and did not receive IV-tPA before MT. To explore whether IV-tPA affected our results, we performed subcohort analyses and observed little influence of IVtPA (Online Figs 2 and 4). Larger sample sizes will be required to further establish relationships among perviousness, clot biology, and MT outcomes in more controlled populations (ie, cases using only 1 type of MT therapy). We also note that the calculation of perviousness is highly dependent on both image coregistration and the neuroradiologist's ability to identify the clot region. We investigated these factors by calculating the MMI for each coregistered case and by calculating inter- and intrauser error for image-derived parameters (perviousness and density from NCCT) in a subset of our data. Our results showed that there was high coregistration accuracy $(\mathrm{MMI}=92.2 \%)$ and low inter- and intrauser calculation error ( $>15 \%$ across dense and nondense clots), though measurement of density on NCCT alone had a much lower error. In the future, automated software platforms for segmenting the clot region in the NCCT and CTA image pairs could make the calculation of perviousness more accurate and reproducible.

\section{CONCLUSIONS}

The goal of this preliminary study was to investigate the relationship between clot perviousness and the biology of the retrieved clot and to test whether perviousness could directly predict FPE. We found that greater perviousness was related to higher $\% \mathrm{FP}$ composition. The biologic composition of the clot was significantly related to FPE, with higher \%FP clots being more difficult to retrieve in the first pass. From ROC analysis, there was only a moderate ability of perviousness to predict FPE. Imaging metrics that more strongly reflect clot biology or multivariate predictive models may be needed to more accurately predict FPE on pretreatment imaging.

\section{ACKNOWLEDGMENTS}

We thank the patients who participated in this study. The authors acknowledge Mohammed Imtiaz, $\mathrm{PhD}$, and Amber Worral, $\mathrm{PhD}$, for histology assistance; Christopher Bigley, MS, and Arvind Ramaswamy for biobanking assistance; and Jennifer L. Gay, CCRP, and Elizabeth Shisler for study protocol management.

Disclosures: Maxim Mokin—UNRELATED: Consultancy: Medtronic, Cerenovus, Canon Medical Systems; Grants/Grants Pending: National Institutes of Health R21NS109575*; Stock/Stock Options: Serenity Medical, Synchron, EndoStream, Vicis. John Kolega - RELATED: Grant: National Science Foundation, National Institutes of Health, Cummings Foundation, Comments: National Science Foundation award \#1746694, National Institutes of Health National-National Institute of Neurological Disorders and Stroke award \#NS115314-0, and Cummings Foundation grant (all with Vincent M. Tutino as Principal Investigator).* John Tomaszewski-OTHER RELATIONSHIPS: I am an investor and consultant for Neurovascular Diagnostics, which is a startup company seeking to model vascular aneurysm progression from neutrophil-expressed gene data. Dr Tutino is a principal in the company. Elad I. Levy_UNRELATED: Board Membership: Stryker, NeXtGen Biologics, MedX Health, Cognition Medical, EndoStream; Consultancy: Claret Medical, GLG Consulting, Guidepoint, Imperative Care, Medtronic, Rebound Therapeutics, StimMed; Employment: University at Buffalo Neurosurgery Inc; Expert Testimony: renders medical/legal opinions as an expert witness; Stock/Stock Options: NeXtGen Biologics, Cognition Medical, Rapid Medical, Claret Medical, Imperative Care, Rebound Therapeutics, StimMed. Jason M. Davies_UNRELATED: Consultancy: Medtronic; Grants/Grants Pending: American Heart Association; Stock/Stock Options: Rist Neurovascular, Cerebrotech Medical Systems. Kenneth V. SnyderRELATED: Grant: Canon Medical Systems, Medtronic, Abbott Vascular ev3; Consulting Fee or Honorarium: Toshiba, Canon Medical Systems, ev3, Medtronic*. Adnan H. Siddiqui-UNRELATED: Consultancy: Amnis Therapeutics, Boston Scientific, Canon Medical Systems, Cerebrotech Medical Systems, Cerenovus, Corindus, EndoStream, Imperative Care, Integra LifeSciences, Medtronic, MicroVention, Minnetronix Neuro, Northwest University, Data and Safety Monitoring Board Chair for the HEAT Trial, Penumbra, Q'Apel Medical, Rapid Medical, Rebound Therapeutics, Serenity Medical, Silk Road Medical, StimMed, Stryker, Three Rivers Medical, VasSol, W.L. Gore \& Associates; Grants/Grants Pending: Coinvestigator: National Institutes of Health/National Institute of Neurological Disorders and Stroke 1R01NS091075, Virtual Intervention of Intracranial Aneurysms; role: Co-Principal Investigator of National Institutes of Health-National Institute of Neurological Disorders and Stroke R21 NS109575-01, Optimizing Approaches to Endovascular Therapy of Acute Ischemic Stroke; Stock/Stock Options: Adona Medical, Amnis Therapeutics, Blinktbi, Boston Scientific (for purchase of Claret Medical), Buffalo Technology Partners, Cardinal Consultants, Cerebrotech Medical Systems, Cognition Medical, EndoStream, Imperative Care, International Medical Distribution Partners, Neurovascular Diagnostics, Q'Apel Medical, Radical Catheter Technologies, Rebound Therapeutics (purchased in 2019 by Integra LifeSciences), Rist Neurovascular, Sense Diagnostics, Serenity Medical, Silk Road Medical, Spinnaker Medical Consultants, StimMed, Synchron, Three Rivers Medical, Vastrax, VICIS, Viseon; Other: National Principal Investigator/Steering Committees: Cerenovus NAPA Trial and ARISE II Trial; Medtronic SWIFT PRIME and SWIFT DIRECT Trials; MicroVention FRED Trial and CONFIDENCE study; MUSC 
POSITIVE Trial; Penumbra 3D Separator Trial, COMPASS Trial, INVEST Trial. Vincent M. Tutino-RELATED: Grant: Clinical and Translational Science Awards Program, Comments: pilot study grant through the University at Buffalo Clinical and Translational Science Institute*; UNRELATED: Employment: Neurovascular Diagnostics, Comments: Cofounder. *Money paid to the institution.

\section{REFERENCES}

1. Santos EM, Marquering HA, Den Blanken MD, et al; MR CLEAN Investigators. Thrombus permeability is associated with improved functional outcome and recanalization in patients with ischemic stroke. Stroke 2016;47:732-41 CrossRef Medline

2. Riedel CH, Zimmermann P, Jensen-Kondering U, et al. The importance of size: successful recanalization by intravenous thrombolysis in acute anterior stroke depends on thrombus length. Stroke 2011;42:1775-77 CrossRef Medline

3. Moftakhar P, English JD, Cooke DL, et al. Density of thrombus on admission CT predicts revascularization efficacy in large vessel occlusion acute ischemic stroke. Stroke 2013;44:243-45 CrossRef Medline

4. Welsh JD, Stalker TJ, Voronov R, et al. A systems approach to hemostasis, 1: the interdependence of thrombus architecture and agonist movements in the gaps between platelets. Blood 2014;124:1808-15 CrossRef Medline

5. Voronov RS, Stalker TJ, Brass LF, et al. Simulation of intrathrombus fluid and solute transport using in vivo clot structures with single platelet resolution. Ann Biomed Eng 2013;41:1297-1307 CrossRef Medline

6. Hashimoto T, Hayakawa M, Funatsu N, et al. Histopathologic analysis of retrieved thrombi associated with successful reperfusion after acute stroke thrombectomy. Stroke 2016;47:3035-37 CrossRef Medline

7. Fitzgerald S, Wang S, Dai D, et al. Orbit image analysis machine learning software can be used for the histological quantification of acute ischemic stroke blood clots. PLoS One 2019;14:e0225841 CrossRef Medline

8. Boeckh-Behrens T, Kleine JF, Zimmer C, et al. Thrombus histology suggests cardioembolic cause in cryptogenic stroke. Stroke 2016;47:186471 CrossRef Medline

9. Marder VJ, Chute DJ, Starkman S, et al. Analysis of thrombi retrieved from cerebral arteries of patients with acute ischemic stroke. Stroke 2006;37:2086-93 CrossRef Medline

10. Sporns PB, Hanning U, Schwindt W, et al. Ischemic stroke: histological thrombus composition and pre-interventional CT attenuation are associated with intervention time and rate of secondary embolism. Cerebrovasc Dis 2017;44:344-50 CrossRef Medline

11. Shin JW, Jeong HS, Kwon HJ, et al. High red blood cell composition in clots is associated with successful recanalization during intra-arterial thrombectomy. PLoS One 2018;13:e197492 CrossRef Medline

12. Borggrefe J, Kottlors J, Mirza M, et al. Differentiation of clot composition using conventional and dual-energy computed tomography. Clin Neuroradiol 2018;28:515-22 CrossRef Medline

13. Zaidat OO, Castonguay AC, Linfante I, et al. First pass effect. Stroke 2018;49:660-66 CrossRef Medline

14. Higashida RT, Furlan AJ; Technology Assessment Committee of the American Society of Interventional and Therapeutic Neuroradiology; Technology Assessment Committee of the Society of Interventional Radiology. Trial design and reporting standards for intra-arterial cerebral thrombolysis for acute ischemic stroke. Stroke 2003;34: e109-37 CrossRef Medline

15. Johnson H, Harris G, Williams K. BRAINSFIT: Mutual Information Rigid Registrations of Whole-Brain 3D Images, Using the Insight Toolkit. https://www.nitrc.org/docman/view.php/52/639/BRAINSFit. pdf. Accessed 08/15/2020

16. Santos EM, Yoo AJ, Beenen LF, et al; MR CLEAN investigators. Observer variability of absolute and relative thrombus density measurements in patients with acute ischemic stroke. Neuroradiology 2016;58:133-39 CrossRef Medline
17. Stritt M, Stalder AK, Vezzali E. Orbit image analysis: an opensource whole slide image analysis tool. PLoS Comput Biol 2020;16: e1007313 CrossRef Medline

18. Santos EM, Dankbaar JW, Treurniet KM, et al. Permeable thrombi are associated with higher intravenous recombinant tissue-type plasminogen activator treatment success in patients with acute ischemic stroke. Stroke 2016;47:2058-65 CrossRef Medline

19. Berndt M, Friedrich B, Maegerlein C, et al. Thrombus permeability in admission computed tomographic imaging indicates stroke pathogenesis based on thrombus histology. Stroke 2018;49:2674-82 CrossRef Medline

20. Benson JC, Fitzgerald ST, Kadirvel R, et al. Clot permeability and histopathology: is a clot's perviousness on CT imaging correlated with its histologic composition? J NeuroIntervent Surg 2020;12:3842 CrossRef Medline

21. Altman DG, Royston P. The cost of dichotomising continuous variables. BMJ 2006;332:1080 CrossRef Medline

22. McDonald MM, Archeval-Lao JM, Cai C, et al. Iodinated contrast does not alter clotting dynamics in acute ischemic stroke as measured by thromboelastography. Stroke 2014;45:462-66 CrossRef Medline

23. Hertig G, Zehnder M, Woloszyk A, et al. Iodixanol as a contrast agent in a fibrin hydrogel for endodontic applications. Front Physiol 2017;8:152 CrossRef Medline

24. Niesten JM, van der Schaaf IC, van Dam L, et al. Histopathologic composition of cerebral thrombi of acute stroke patients is correlated with stroke subtype and thrombus attenuation. PLoS One 2014;9:e88882 CrossRef Medline

25. Kirchhof K, Welzel T, Mecke C, et al. Differentiation of white, mixed, and red thrombi: value of $\mathrm{CT}$ in estimation of the prognosis of thrombolysis phantom study. Radiology 2003;228:126-30 CrossRef Medline

26. Gersh KC, Nagaswami C, Weisel JW. Fibrin network structure and clot mechanical properties are altered by incorporation of erythrocytes. Thromb Haemost 2009;102:1169-75 CrossRef Medline

27. Maekawa K, Shibata M, Nakajima H, et al. Erythrocyte-rich thrombus is associated with reduced number of maneuvers and procedure time in patients with acute ischemic stroke undergoing mechanical thrombectomy. Cerebrovasc Dis Extra 2018;8:39-49 CrossRef Medline

28. Gunning GM, McArdle K, Mirza M, et al. Clot friction variation with fibrin content; implications for resistance to thrombectomy. $J$ NeuroIntervent Surg 2018;10:34-38 CrossRef Medline

29. Byun JS, Nicholson P, Hilditch CA, et al. Thrombus perviousness is not associated with first-pass revascularization using stent retrievers. Interv Neuroradiol 2019;25:285-90 CrossRef Medline

30. Bernava G, Rosi A, Boto J, et al. Direct thromboaspiration efficacy for mechanical thrombectomy is related to the angle of interaction between the aspiration catheter and the clot. J Neuronterv Surg 2020;12:396-400 CrossRef Medline

31. Qiu W, Kuang H, Nair J, et al. Radiomics-based intracranial thrombus features on CT and CTA predict recanalization with intravenous alteplase in patients with acute ischemic stroke. AJNR Am Neuroradiol 2019;40:39-44 CrossRef Medline

32. Boeckh-Behrens T, Schubert M, Förschler A, et al. The impact of histological clot composition in embolic stroke. Clin Neuroradio 2016;26:189-97 CrossRef Medline

33. Jagani M, Kallmes DF, Brinjikji W. Correlation between clot density and recanalization success or stroke etiology in acute ischemic stroke patients. Interv Neuroradiol 2017;23:274-78 CrossRef Medline

34. Dutra BG, Tolhuisen ML, Alves $\mathrm{H}$, et al; MR CLEAN Registry Investigators. Thrombus imaging characteristics and outcomes in acute ischemic stroke patients undergoing endovascular treatment. Stroke 2019;50:2057-64 CrossRef Medline

35. Mokin M, Morr S, Natarajan SK, et al. Thrombus density predicts successful recanalization with Solitaire stent retriever thrombectomy in acute ischemic stroke. J Neurointerv Surg 2015;7:104-07 CrossRef Medline 